Revista de Psicología y Educación / Journal of Psychology and Education, 2019, 14(2), $112-123$ (www.rpye.es) Doi: https://doi.org/10.23923/rpye2019.02.176

ISSN: $1699-9517$

\title{
Relación entre género, resiliencia y autoconcepto académico y social en la adolescencia
}

\author{
Cristina Pinel-Martínez*, María del Carmen Pérez-Fuentes y José Juan Carrión-Martínez \\ Universidad de Almería, Almería, España
}

\begin{abstract}
Resumen: La adolescencia se caracteriza por la sucesión de cambios biofisiológicos, sociales, emocionales, psicológicos e intelectuales, que determinan la construcción del autoconcepto y de la resiliencia, la cual sirve de base para conllevar las adversidades que encontrarán a lo largo de la vida. Los principales objetivos de este trabajo son describir los niveles de resiliencia, autoconcepto social y académico, y determinar la correlación entre resiliencia, autoconcepto social y académico. Este estudio transversal de carácter descriptivo-exploratorio se ha llevado a cabo con una muestra de 60 adolescentes, con una edad media de $M=13,02$ (D.T: 0,911) y un rango de edad entre 11 y 14 años. Se les aplicó la escala CD-RISC y el test AF5. El análisis de los datos se llevó a cabo a través del programa estadístico SPSS en su versión 23.0. Los resultados mostraron unos niveles altos de resiliencia, autoconcepto social y académico entre los jóvenes. Se observaron correlaciones positivas entre la resiliencia, el autoconcepto académico y el social. La relación existente entre los niveles de autoconcepto y resiliencia puede servir como punto de apoyo sobre el que impulsar su investigación para determinar las causas de sus posibles variaciones a lo largo del ciclo vital.
\end{abstract}

Palabras clave: Resiliencia, Género, Autoconcepto, Adolescencia, Correlación.

Relationship between gender, resilience, academic and social self-concept in adolescence

Abstract: Adolescent years are filled with biophysiological, social, emotional, psychological and intellectual changes that determine the unfolding self-concept and resilience, which serves as the bases for coping with the adversities they may face in their daily life. The main objectives of this study are to identify the levels of resilience, social and academic self-concept in relation to teenagers' gender and to determine the correlation between resilience, social and academic self-concept of adolescents. This descriptiveexploratory and cross-sectional rehearsal has been implemented in 60 adolescents with an average age of $M=13.02$ (S.D: 0.911 ) and an age range between 11 y 14 years old, who correctly completed the CD-RISC and the AF5 tests. Data analysis was performed using the Statistical Package SPSS 23.0. Results brought to light high levels of general resilience, social self-concept and academic self-concept levels. Positive correlations were observed between resilience and academic self-concept and social self-concept, as well as between the two types of self-concept. The relationship between levels of self-concept and resilience can serve as keystone to keep on exploring these constructs to determine the causes of their possible variations throughout the life cycle.

Keywords: Resilience, Gender, Self-concept, Adolescence, Correlation.

El ser humano se caracteriza, entre otras cualidades, por su necesidad de encontrar el sentido que brindan a su existencia las diferentes situaciones que se presentan a lo

Recibido: 17/09/2018 - Aceptado: 16/01/2019 - Avance online: 22/02/2019

*Correspondencia: Cristina Pinel Martínez.

Universidad de Almería, Almería, España

C.P: 04120 , Almería, España.

E-mail: cpm467@ual.es

Pinel-Martínez, C., Pérez-Fuentes, M. C. y Carrión-Martínez, J. J. (Avance Online). Relación entre género, resiliencia y autoconcepto académico y social en la adolescencia. Relationship between gender, resilience, academic and social self-concept in adolescence. Journal of

Psychology and Education, 14(2), 112-123, hittps://doi.org/10.23923/rpye2019.02.176 largo de su vida, a veces siendo realidades sin trascendencia y otras con un gran peso que marcan y condicionan permanentemente a cada persona (Rincón, 2016). En la mayoría de ocasiones, las circunstancias de vida que definen a cada individuo, ya se vivencien positiva o negativamente, no están predeterminadas en tiempo o espacio, sino que pueden acaecer de manera fortuita $y$

1699-9517/@ 2019 Asociación Científica de Psicología y Educación (ACIPE). Publicado por Consejo General de Colegios Oficiales de Psicólogos, España. Este es un artículo Open Access bajo la CC BY-NC-ND licencia (http://creativecommons.org/licencias/bync-nd/4.0/ 
esporádica o incluso repetirse y prolongarse a lo lago de diferentes periodos vitales (Coetzee, Ebersöhn, Ferreira y Moen, 2017). Ésta dinámica es la principal precursora del avance y desarrollo del ser humano (Kong, Wang, Hu y Jia, 2015), suponiendo un cambio hacia la mejora íntegra de la persona si esta es capaz de aprovechar cada escenario vivido, o un empeoramiento de las condiciones de vida actuales y futuras si la persona no es capaz de afrontar cada circunstancia satisfactoriamente (Ganotice, Yeung, Beguina y Villarosa, 2016).

Tal y como se puede observar, en este proceso de desarrollo y cambio constante se deben incluir tanto los periodos de esplendor como aquellos momentos bajos que se consideran estresantes. Sin embargo, siguiendo las palabras de Rincón (2016) las culturas occidentales, entre las que incluimos la nuestra, tienden a rehusar las situaciones anómalas que pueden provocar en el individuo daños o perjuicios proporcionando facilidades para asegurar el bienestar social y la salud física y mental, evitando en cierto modo que las personas tengan que enfrentarse a esos escenarios adversos. Aun así, el acontecimiento de dificultades e infortunios es inevitable, constituyendo una parte fundamental del propio ciclo vital, siendo su aceptación de gran importancia en el desarrollo de capacidades que permitan enfrentar, asimilar y superar las circunstancias del día a día (García, Ríos, Carrillo y Sabuco, 2018).

Los individuos que poseen y ponen en práctica este tipo de habilidades, obteniendo un aprendizaje y desarrollo positivo a través de situaciones consideradas desestabilizantes, son aquellos que poseen lo que comúnmente se llama un "perfil resiliente" (Kim y Lee, 2018). La resiliencia está definida como la capacidad de superar situaciones adversas, independientemente del grado de gravedad con el que las percibe cada individuo, que permite salir de ellas fortalecido y de manera airosa (Bouillet, Ivanec y Miljević, 2014; Campos y González, 2018; Rincón, 2016; Rodríguez, Ramos, Martínez y Rey, 2018). Las personas resilientes son capaces de tomar situaciones consideradas perjudiciales como una base para el crecimiento personal y el autoaprendizaje (Richards, Levesque, Templin y Graber 2016).

El estudio del desarrollo de características resilientes suele centrarse en personas pertenecientes a grupos considerados de riesgo que, en contra de todo pronóstico, no han seguido un desarrollo anómalo (Muñoz, 2016 ; Pinto, 2014 ; Costa, Barontini, Forcada, Carrizo y Almada, 2010). Sin embargo, esta capacidad puede ser aprendida e internalizada a cualquier edad e independientemente de la situación personal (Pintado y Cruz, 2017). Aun así, debemos tener en cuenta que no todas las personas pueden llegar a desarrollarla o del mismo modo, poniendo de manifiesto la influencia que algunos factores, como el género, la educación recibida o la edad, pueden llegar a ejercer en su adquisición (Vinaccia, Quiceno y Moreno, 2007).

Si nos fijamos en la edad, aunque esta capacidad tiene tendencia natural a verse reforzada con el trascurso de los años, existe un reconocimiento generalizado sobre la importancia de desarrollar la resiliencia en edades tempranas debido a los múltiples beneficios que puede aportar desde la primera infancia si forma parte del proceso madurativo de la persona, ya que puede ayudar reafirmar la identidad y aumentar la autoconfianza o la seguridad (Coppari, Barcelata, Bagnoli y Codas, 2018; Fínez y Morán, 2017; Sampedro, Alvarez y Begega, 2018). Otros autores afirman que puede mejorar el proceso de aprendizaje, fomentar un desempeño escolar satisfactorio (Acevedo y Restrepo, 2012) y evitar problemas mentales, conductas disruptivas o comportamientos disfuncionales tales como bullying, consumo de drogas o violencia (Esturgó y Sala, 2010; González y Valdez, 2015).

Con este fin y gracias a la necesidad de crear personas resilientes desde la infancia, es cada vez más frecuente su estudio en diferentes etapas educativas, así como su inclusión en el proceso de enseñanza-aprendizaje, ya sea por medio de implementaciones a través de maestros y profesores o "tutores de resiliencia" (García y Domínguez, 2013; Pinto, 2014) o de determinar los factores delimitantes en el 
desarrollo psicosocial durante estas edades (Pintado y Cruz, 2017; Ramírez y Castro, 2018). Dentro de la población estudiantil, destacamos a los alumnos de educación secundaria por pertenecer a un sector en los que se dan grandes cambios fisiológicos y psicológicos, siendo la incapacidad de los alumnos para enfrentarse a dichos cambios de manera autosuficiente la causa principal de diversos desequilibrios (Salum, Marín y Reyes, 2011). Igualmente, debemos tener en cuenta el género de los estudiantes, ya que aunque nos encontramos en una sociedad cada vez más igualitaria, las propias diferencias físicas y biológicas de cada género influyen en su desarrollo, sobre todo en la etapa de la adolescencia (Finez y Morán, 2014). En estudios como los realizados por Finez y Morán (2014), las alumnas tendían a tener menores niveles de resiliencia que sus semejantes varones por ser ellos los que más capacidad demostraban para enfrentarse a los contratiempos y los que más factores protectores adquirían. Otros estudios como los realizados por Villalta y Saavedra (2012) mostraban resultados contradictorios, teniendo las chicas unas medias más elevadas que los hombres aunque sin existir significatividad en estas diferencias.

Además de los cambios que se pueden llegar a producir como consecuencia del género o la edad, debemos tener en cuenta que la resiliencia es el producto de la adquisición de factores protectores, entre otros, el pensamiento crítico, la creatividad (Muñoz, 2016), el humor, la autoeficacia (Fínez y Morán, 2016), el sentimiento de control, el compromiso o la aceptación del miedo al fracaso (Fínez y Morán, 2014). Estos factores protectores están directamente ligados con el desarrollo y la percepción del "yo" en el alumno, refiriéndonos no solo a la persona en sí misma junto con sus cualidades, sino también a la capacidad de regular su conducta respecto aquellas cosas que le rodean, las situaciones que experimenta y los objetos y elementos que cada estudiante considera como suyos (Andrade, Duffay, Ortega, Ramirez y Carvajal, 2017; Himmel, 2002). Esta función de regulación se da gracias a la autoevaluación y la autoconciencia, dos procesos que permiten a cada individuo conocer sus propias capacidades, dando forma al llamado autoconcepto, el cual es a su vez está orientado hacia el bienestar y el propósito de superación (Bernal y Gálvez, 2017; Valenzuela y López, 2015) Por tanto, para el desarrollo de la resiliencia en la etapa educativa, sería importante conocer los niveles que presentan los estudiantes de autoconcepto.

El autoconcepto está considerado como la definición que cada individuo crea de su persona a partir de factores externos e internos, es decir, una serie de creencias sobre lo que las personas consideran "verdaderas respecto a sí mismos" (Padilla, García, y Suárez, 2008; Valenzuela y López, 2015). Este cambia a lo largo de los años dependiendo de las experiencias vividas por cada uno y se vincula, al igual que la resiliencia, con la edad y el género de los estudiantes, creando una relación directa y positiva respecto a los niveles de resiliencia (González y Valdez, 2015). Según estudios como los realizados por Gonzáles, Huamán, Cubas y Visalot (2018) el 28,7\% de los estudiantes manifiestan un autoconcepto positivo y unos niveles de resiliencia media y el $27,3 \%$ de los estudiantes con autoconcepto medio tenían niveles altos de resiliencia. Rodríguez et al. (2018) mostraron que cuando los niveles de resiliencia y de autoconcepto se presentaban altos, resultaban ser predictores directamente proporcionales a la implicación escolar y el desarrollo de habilidades para la vida.

Aunque en los inicios del estudio del autoconcepto éste estuvo considerado como un constructo unidimensional, diversos autores lo dividen en diferentes dimensiones que aluden a dichos factores internos y externos mencionados anteriormente. Siguiendo el ejemplo de autores como Fínez y Morán, (2014), quienes toman la división realizada por James en 1985, el autoconcepto costa de "yo material", "yo social" y "yo espiritual". Autores más recientes fragmentan en autoconcepto en cinco factores en los que se puede observar la influencia de las primeras definiciones: social, académico, familiar, físico 
y espiritual, haciendo de este constructo un elemento multidimensional (Bernal y Gálvez, 2017; Fuentes, García, Gracía y Lila, 2011 ; Garaigordobil, Durá y Pérez, 2005; Salum et al., 2011).

Si bien es cierto que todas las dimensiones afectan de una manera $u$ otra en la progresión del individuo, el autoconcepto social y el autoconcepto académico están especialmente ligados al crecimiento de los alumnos que cursan la etapa de Educación Secundaria Obligatoria (Costa y Tabernero, 2012). Durante este periodo, la influencia que ejercen las opiniones de su grupo de iguales es mayor que en otras etapas, afectando al desarrollo de su autoconcepto de una manera más significativa (Tabernero, Serrano y Mérida, 2017). Del mismo modo, el entorno académico es uno de los contextos que cobra más importancia en el desarrollo biopsicosocial de cada individuo, ya que pasan gran parte de su vida en este escenario. De esta manera, es la base principal para la construcción de la percepción de su rol como estudiante y el pilar en el que se sustenta el aprendizaje que le llevará a relacionarse de manera congruente con su entorno social (Sosa, Sánchez y Guerrero, 2016). Si hacemos alusión al género, estas dos variables de autoconcepto suelen presentar diferencias entre los niños y las niñas en los estudios realizados. En ciertas investigaciones, los varones son los que muestran mayores niveles de autoconcepto social (Reynoso, Caldera, de la Torre, Martínez y Macías, 2018) y las mujeres puntúan más alto en los niveles de autoconcepto académico (Sosa et al., 2016). Sin embargo, en otros estudios son las mujeres las que presentan niveles más elevados tanto en académico como en el social (Padilla et al., 2010), o incluso ambos géneros mantienen una tendencia alta y equilibrada (Fernández, Goñi, Rodríguez y Goñi, 2017).

Como podemos observar, existe una controversia respecto a los resultados de las investigaciones realizadas en los últimos años sobre las diferencias en los niveles de resiliencia y el autoconcepto social y académico. Esta diferencias de opiniones se dan sobre todo dentro del grupo social en el que se incluyen los estudiantes, así como es las posibles variaciones dependiendo del género del alumnado. Por esta razón y tras esta revisión de la literatura, los objetivos del actual trabajo serán los de identificar y describir los niveles de resiliencia, autoconcepto social y académico en adolescentes; determinar la relación entre el género y los niveles de resiliencia y de autoconcepto social y académico en adolescentes; y definir la correlación entre resiliencia, autoconcepto social y académico de adolescentes.

\section{MÉTODO}

\section{PARTICIPANTES}

Nos encontramos ante un trabajo de corte transversal, de carácter descriptivo y exploratorio, el cual ha sido llevado a cabo sobre una muestra de 60 adolescentes pertenecientes a diferentes institutos localizados en el centro y suroeste de la ciudad de Granada (España). El 61,7\% $(n=37)$ de la muestra estaba compuesta por chicos, mientras que el $38,3 \% \quad(n=23)$ de participantes restantes pertenecían al sector femenino, con una edad media de $M=13,02$ (entre 11 y 14 años, D.T: 0,911).

\section{INSTRUMENTOS DE MEDIDA}

Para la recogida de datos sociodemográficos, se utilizó una hoja de autorregistro con la que se determinaron las variables de edad (respuesta libre) y género (masculino/ femenino) de los encuestados. Al mismo tiempo, se presentó la Escala de resiliencia CD-RISC (Connor-DavidsonReslienceScale), propuesta por Connor y Davidson en 2003. En este caso, nos encontramos ante un test con 25 preguntas cuyas respuestas se encuentran predispuestas en una escala tipo Likert del 1 al 4, correspondientes consecutivamente a "Nada de acuerdo", "Casi de acuerdo", "De acuerdo" y "Totalmente de acuerdo". Sus ítems se dividen de la siguiente manera: Locus de control y compromiso $(21,4,19,22,17)$, Desafío de conducta orientada a la Acción (2, 13), Autoeficacia y Resistencia al Malestar (7, 
$10,16,24,11,5,15,18,25)$, Optimismo y Adaptación a Situaciones Estresantes (1, 6, 8, $12,14)$ y Espiritualidad $(3,9,20)$. Aunque con este test se da la posibilidad de poder medir por separado cada una de las dimensiones, en este estudio se ha decidido utilizarlas en conjunto para la medición de la resiliencia en general, tal y como suelen hacer la mayoría de investigaciones en este campo (Díaz y Barra, 2017; Richards et al., 2016; Villalta y Saavedra, 2010). Este test se contabiliza con un mínimo de 25 puntos y un máximo de 100, siendo mayor el nivel de resiliencia cuanto más alta es la puntuación final. El índice de fiabilidad que obtuvieron los autores en su investigación fue de $\alpha=89$, encontrándose una fiabilidad con un valor similar en este estudio con un $\alpha=, 876$.

Para la obtención de datos sobre el autoconcepto, se implementó el Test de Autoconcepto Forma 5 (AF5), presentado por García y Musitu en 1999. Para realizarlo, se proporcionó una escala del 1 al 5 donde 1 era "nunca", 2 "Pocas veces", 3 "Algunas veces", 4 "Muchas veces" y 5 "Siempre". El test se compone de 30 preguntas, las cuales se encuentran divididas en cinco tipos de autoconcepto. En este caso, se han utilizado las dimensiones de autoconcepto académico y autoconcepto social por ser, como se ha explicado con anterioridad, dos de los constructos relacionados con los contextos con los que más contacto tienen los adolescentes durante estas etapas y por tanto, los que pueden obtener un mayor desarrollo en este periodo (Padilla et al., 2010; Sosa, Sánchez y Guerrero, 2016). El estudio realizado por los autores, obtuvo una fiabilidad de $\alpha=, 815$. En el presente estudio, la fiabilidad tiene un valor menor, pero igualmente alto $(\alpha=, 788)$. Respecto a las dimensiones, el autoconcepto académico presentó una fiabilidad de $\alpha=, 766$; mientras que el autoconcepto social se obtuvo de $\alpha=, 707$. Las preguntas quedan divididas en Autoconcepto Académico (1, 6, $11,16,21,26)$ y Autoconcepto Social $(2,7$, $12,17,22,27)$, perteneciendo la suma final de las respuestas de todos los ítems del test al autoconcepto global que posee la persona (autoconceptos social, académico, emocional, familiar y físico). A mayor puntuación en cada una de las dimensiones, mayor el autoconcepto del alumno. Se ha utilizado los niveles de autoconcepto general de la tabla 1 creada por los autores como guía, para poder discernir entre niveles bajos, medios y altos de autoconcepto general (Salum et al., 2011 ). A partir de la misma, se ha compuesto una columna propia en la misma tabla para poder determinar los niveles de autoconcepto en cada una de las dimensiones de forma individual (Dimensiones).

\section{Tabla $]$}

Niveles de autoconcepto: Tabla original (general) y de creación propia (Dimensiones)

\begin{tabular}{|c|c|c|}
\hline $\begin{array}{c}\text { Niveles de } \\
\text { autoconcepto }\end{array}$ & General & Dimensiones \\
\hline Autoconcepto Bajo & De 30 a 69 & De 6 a 13 \\
Autoconcepto Medio & De 70 a 109 & De 14 a 21 \\
Autoconcepto Alto & De 110 a 150 & De 22 a 30 \\
\hline
\end{tabular}

\section{PROCEDIMIENTO}

Antes de comenzar con la recogida de datos, se concertó una reunión con los directores y los tutores de los centros implicados, explicando el objetivo, el carácter del estudio y el procedimiento que se iba a llevar a cabo, pidiendo su participación. Una vez aceptaron la petición y al encontrarnos ante una muestra compuesta por menores de edad, se proporcionó la información necesaria por escrito a los participantes y a sus tutores legales, así como un consentimiento firmado de los tutores legales tendrían que firmar en el que se hablaba del carácter voluntario y anónimo de la investigación, cumpliendo así con los requisitos éticos de la Declaración de Helsinki (1975). Se determinaron dos días para cumplimentar el test de forma voluntaria en horario escolar, con la finalidad de obtener el mayor número de participaciones posibles. Por último, se pasó a la recogida de los test para su posterior análisis. La aplicación del test se llevó a cabo de manera colectiva, completando cada alumno individualmente 
su test y preguntando las posibles dudas al investigador principal, utilizando para ello los diez primeros minutos de las clases de aquellos tutores escolares que nos dieron permiso para usar ese tiempo.

En un principio, se consiguieron recoger 135 test entre los diferentes centros, de los cuales solo 60 de ellos estaban completados correctamente, de manera que se decidió proceder solo con los completados en su totalidad para evitar resultados alejados de la realidad. El responsable principal de la investigación se encontraba presente durante todo el proceso de realización del test, el cual se encarga de guardar los datos de manera confidencial. Solo se aceptaron los test de aquellos participantes que se encontraban en el momento de la recogida de datos y de aquellos que no poseyesen ninguna discapacidad psíquica que les impidiese completar los cuestionarios de manera fidedigna. Igualmente, tampoco se aceptaron aquellos test en los que los estudiantes no presentasen, en el momento de la realización de los cuestionarios, el consentimiento firmado de los tutores legales que se había expedido con anterioridad, los cuales se separaron de los test para asegurar la confidencialidad y el anonimato.

\section{ANÁLISIS DE DATOS}

En la interpretación de los datos y en la comparación de las diferentes variables, se ha utilizado el programa estadístico SPSS versión 23.0 en el que se han analizado los datos para realizar el estudio descriptivo
(Medias y frecuencias) y el correlacional. Dado el tamaño de la muestra y tras rechazar la hipótesis de normalidad gracias a la prueba de Kolgomorov-Smirnov, se utilizó el test estadístico no paramétrico $U$ de MannWhitney para dos muestras independientes en las comparaciones de los dos grupos de género, resiliencia, autoconcepto social y autoconcepto académico. Así mismo, se realizó una prueba de análisis de correlación de Spearman para el estudio de la fuerza y la dirección de la relación entre las variables dependientes del estudio.

\section{RESULTADOS}

Tal y como se puede observar en la Tabla 2 (Estadísticos Descriptivos de las principales variables), los datos descriptivos más destacables son las medias de los constructos, las cuales se muestran altas tanto en la variable de resiliencia como en las dos dimensiones de autoconcepto. En el caso de la distribución de los participantes en cuanto a los niveles de resiliencia, la gran mayoría de estos se encuentran dentro del tramo con altos niveles de resiliencia y autoconcepto. Basándonos en los resultados de la Tabla 2, solo una pequeña parte de los participantes tenían unos niveles bajos de autoconcepto académico, mientras que no se encontraron estudiantes que tuvieran niveles bajos de resiliencia o de autoconcepto social. Todos aquellos que no mostraron unos niveles altos de resiliencia o de autoconcepto social, se situaron en los escalones correspondientes a los niveles medios.

Tabla 2

Estadísticos Descriptivos de las principales variables

\begin{tabular}{|c|c|c|c|c|c|c|c|}
\hline \multirow{2}{*}{ Estadísticos descriptivos } & \multirow{2}{*}{ Mín. } & \multirow{2}{*}{ Máx. } & \multirow{2}{*}{$\bar{x}$} & \multirow{2}{*}{ D.T. } & \multicolumn{3}{|c|}{ Niveles porcentuales } \\
\hline & & & & & Bajo & Medio & Alto \\
\hline Resiliencia & 2 & 3,8 & 3,12 & 0,43 & $0 \%(n=0)$ & $35 \%(n=21)$ & $65 \%(n=39)$ \\
\hline Autoconcepto Social & 2,83 & 4,83 & 3,66 & 0,44 & $0 \%(n=0)$ & $45 \%(n=27)$ & $55 \%(n=33)$ \\
\hline Autoconcepto Académico & 2 & 5 & 3,99 & 0,68 & $1,7 \%(n=1)$ & $21,7 \%(n=13)$ & $76,7 \%(n=46)$ \\
\hline
\end{tabular}


Tabla 3

Género por resiliencia, autoconcepto social y autoconcepto académico

\begin{tabular}{|c|c|c|c|c|c|}
\hline \multicolumn{2}{|c|}{$\begin{array}{l}\text { Género por autoconcepto académico, } \\
\text { autoconcepto social y resiliencia }\end{array}$} & Rango promedio & Umw & z & $\mathrm{p}$ \\
\hline Resilencia & $\begin{array}{l}\text { Masculino } \\
\text { Femenino }\end{array}$ & $\begin{array}{l}31,11 \\
29,52\end{array}$ & 403 & $-0,342$ & ,732 \\
\hline Autoconcepto Social & $\begin{array}{l}\text { Masculino } \\
\text { Femenino }\end{array}$ & $\begin{array}{l}29,36 \\
32,33\end{array}$ & 383 & $-0,65$ &, 516 \\
\hline $\begin{array}{l}\text { Autoconcepto } \\
\text { Académico }\end{array}$ & $\begin{array}{l}\text { Masculino } \\
\text { Femenino }\end{array}$ & $\begin{array}{l}28,54 \\
33,64\end{array}$ & 353 & $-1,11$ & 267 \\
\hline
\end{tabular}

Tabla 4

Correlación Bivariada de Spearman para las variables de autoconcepto académico, social y resiliencia.

\begin{tabular}{|c|c|c|}
\hline $\begin{array}{c}\text { Correlación } \\
\text { entre resiliencia, } \\
\text { autoconcepto social } \\
\text { y autoconcepto } \\
\text { académico }\end{array}$ & Autoconcepto & Dimensiones \\
\hline Social & Autoconcepto &, $625^{\star *}$ \\
\hline Académico & & , 318** \\
\hline
\end{tabular}

Tal y como se presentan los resultados de la Tabla 3 (Género por resiliencia, autoconcepto social y autoconcepto académico), no existen diferencias estadísticamente significativas ( $p$ $<$,05), entre los niveles de autoconcepto académico, autoconcepto social y resiliencia en comparación con el género de los estudiantes.

En el análisis correlacional se puede observar una relación positiva, significativa y alta entre las variables de resiliencia y autoconcepto académico al nivel de ,05. De esta manera, la resiliencia se encuentra positivamente relacionada conel Autoconcepto Social. Por otra parte, la resiliencia también ha mostrado una relación positiva, pero esta vez moderada, con el autoconcepto social, al nivel de ,01. La relación que se mostró entre las dos dimensiones de autoconcepto fueron moderas y positivas en el nivel, 05 .

\section{DISCUSIÓN}

En este estudio, se puede encontrar que la media de la resiliencia se mantiene en valores bastantes elevados. Fínez y Morán (2014) toman la resiliencia desde el punto de vista académico, presentando medias igualmente altas y comparándolas con los niveles de agotamiento de los estudiantes, los cuales suelen ser bajos. Otros estudios muestran resultados contradictorios, con bajos niveles de resiliencia en niños de educación primaria. Sin embargo, estos estudios se centran en colegios en desventaja social y económica, donde los niños no han desarrollado factores de protección internos (autoeficacia, aprendizaje, identidad, etc.) ni externos (apoyo familiar, confianza en las personas, afectividad, etc.) (Campos y González, 2018). Estos datos dan a entender la importancia de mantener y enseñar al alumnado a cómo mantener los niveles de resiliencia altos, evitando así el agotamiento académico y mejorando la propia percepción del alumnado de sus habilidades académicas y para la vida (Gonzáles, Huamán, cubas y Visalot, 2018).

Respecto al autoconcepto, se ha considerado en la presente investigación priorizar el estudio del Autoconcepto Social y del Autoconcepto Académico frente al resto de dimensiones de este constructo. Aunque existen varias investigaciones que estudian el autoconcepto, incluyendo el físico, emocional y familiar (Padilla et al., 2010), diversos autores se encuentran de acuerdo 
en que la falta de autoconcepto social y académico están directamente relacionados con múltiples dificultades en estudiantes en edades entre 12 y 20 años, como por ejemplo, una inadecuada percepción del rol de estudiante o incapacidad para mantener relaciones interpersonales (Bernal y Gálvez, 2017; Fernández, Goñi, Rodríguez y Goñi, 2017; Sosa, Sánchez y Guerrero, 2016). De acuerdo con los resultados obtenidos en este trabajo, las medias de Autoconcepto Social y Autoconcepto Académico se mantienen altas en el alumnado de educación secundaria. Estos resultados se repiten en investigaciones tales como las llevadas a cabo por Padilla et al. (2010) o Hernández, Hernández y López (2017) las cuales afirman que los estudiantes, sobre todos aquellos que se encuentran en las etapas de primaria y de secundaria, mantienen unos niveles de autoconcepto altos (al igual que pasa con los de resiliencia), debido al apoyo familiar, al soporte que los jóvenes suelen encontrar en sus semejantes y las propias expectativas de futuro que los mueven a mejorar académicamente en el presente (Himmel, 2002; Rodríguez, Ramos, Martínez y Rey, 2018).

Siguiendo en la línea del autoconcepto, más de tres cuartos de los estudiantes se encontraban por encima de la media o dentro de los valores normales de Autoconcepto Académico. No se observaron participantes con niveles preocupantemente bajos en el Autoconcepto Social o en los niveles de Resiliencia. En términos generales, son pocos los casos que se dan de estudiantes con baja autoestima o con niveles de resiliencia por debajo de la media. Como hemos mencionado anteriormente, los estudiantes que pertenecen a este grupo de personas suelen ser alumnos con algún tipo de discapacidad, dificultad, problema físico o psíquico, o alumnado en situación de desventaja social o económica (Andrade, Duffay, Ortega, Ramírez y Carvajal, 2017; Tabernero, Serrano y Mérida, 2017)

En cuanto a la comparación de los niveles de resiliencia respecto al género de los participantes, los resultados encontrados no mostraron diferencias significativas entre niños y niñas. La falta de significatividad hace que los resultados no apoyen los de otros estudios en los que si se encuentran diferencias significativas, como por ejemplo, la investigación realizada por Pintado y Cruz (2017) o Ramírez y Castro (2018) donde se encontró una tendencia natural en los hombres a tener mayores niveles de resiliencia que las mujeres, independientemente de la situación en la que estos se encuentren. En nuestro caso, la aproximación que tienen los niveles de resiliencia entre niños y niñas se puede deber a la zona geográfica en la que se encuentra el colegio. Este alumnado vive en una sociedad que, por una parte, da gran importancia al equilibrio social y el bienestar presente y futuro de los individuos que la componen y, por otra, en la que cada vez es más frecuente encontrar una igualdad de condiciones en mujeres y hombres, ofreciéndoles un trato similar y las mismas oportunidades para desarrollar esta capacidad (Rincón, 2016)

En la comparación de medias respecto al género y las variables de autoconcepto, las diferencias encontradas en este estudio tampoco fueron significativas. Podemos encontrar una gran variedad de resultados cuando hablamos de género y autoconcepto. Por ejemplo, en la investigación llevada a cabo por Garaigordobil, Durá y Pérez (2005), aunque las mujeres puntúan por encima de los hombres en lo que refiere a Autoconcepto Académico, se puede encontrar una similitud con los resultados presentados en este estudio en cuanto autoconcepto social, ya que no los autores tampoco encontraron diferencias en esta dimensión. En otros estudios como el de Padilla, García y Suárez (2010), si encontraban una diferencia significativa a favor de las mujeres en el Autoconcepto Académico, mientras que los hombres presentaban mayores medias en el Autoconcepto Social. Esta discrepancia en los resultados de investigación hallados en torno al autoconcepto, demuestra la importancia de continuar trabajando sobre este constructo, teniendo en cuenta diferentes variables relacionadas con los estudiantes para determinar posibles causantes de este tipo de variaciones en poblaciones tan similares.

En cuanto el análisis correlacional entre 
las diferentes variables, este ha mostrado una relación positiva y moderadamente alta, entre la resiliencia y la dimensión del autoconcepto académico. Entre la resiliencia y el autoconcepto social, aunque también existe una relación positiva, está se muestra a unos niveles moderados, situación que se repite en la relación entre las dimensiones del autoconcepto. Estos resultados son similares a los que muestran investigaciones como la llevada a cabo por Fínez y Morán, (2014), en las que el autoconcepto y la resiliencia presentan una correlación igualmente positiva, aunque moderada, con una correlación de $r=$,386. Otros autores, exponen relaciones significativas similares a las mostradas en este estudio en referencia al Autoconcepto Social y Académico, con una relación igualmente positiva, aunque en este caso sean fuertes (Fuentes, García, Gracia y Lila, 2011). Los alumnos resilientes son capaces de crear una imagen más positiva sobre sí mismos, lo que a su vez genera menos desconfianza en sus propias capacidades y mayor destreza para superar situaciones adversas, lo que podría explicar estas relaciones que se muestran en los resultados (Fínez y Morán, 2014). Sin embargo, debemos tener en cuenta la posibilidad de que esta relación se pueda ver influenciada por una tercera variable no tenida en cuenta en el presente estudio en el análisis correlacional, como por ejemplo, la edad, la localización del centro, la personalidad, o la situación familiar del alumnado (Acevedo y Restrepo, 2012; Campos y González, 2018). Estos factores podrían suponer una variación en el grado de relación o incluso en la popia causalidad entre la resiliencia y el autoconcepto en caso de que se introduciesen en el estudio estas y otras vaiables no controladas.

Se considera necesario hablar de las limitaciones presentes en este estudio, de manera que puedan ser tenidas en cuenta en futuras investigaciones. La muestra que se ha utilizado es pequeña, estando compuesta por un total de 60 estudiantes, lo que podría aumentar el riesgo de error en los resultados. Este número se debe, por una parte, a las dificultades que se han encontrado al realizar el test y por otra, a la necesidad de que todos los niños traigan el consentimiento firmado de sus tutores legales en los días en los que se procede aplicar los cuestionarios. Aunque los cuestionarios elegidos estaban validados para las edades con las que se ha trabajado, los alumnos han presentado dudas en algunos de los apartados, cuya redacción podía dar lugar a ambigüedades. Esta dificultad se ha intentado suplir a través de las explicaciones dadas por el investigador principal. Aun así, más de la mitad de los niños dejaron ítems sin contestar o con varias respuestas, lo que invalidaba dichos test, dejándonos con una población más pequeña de la deseada. Sería por tanto de interés y una mejora en el proceso, la creación y validación de un test que realmente se adapte a este sector educativo, evitando preguntas ambiguas y extensas que puedan confundir a alumnado.

Por otra parte, los resultados de este estudio muestran una relación entre estos dos constructos, desvelando la importancia de seguir esta línea de investigación para poder establecer relaciones más precisas. En este caso, se podían utilizar poblaciones más extensas, así como otras variables de diversa índole (personal, académica, social, etc.), con las que se pueda determinar las verdaderas causas y dirección de dicha relación y hacer un perfil más preciso.

Tal y como se puede observar, el autoconcepto y la resiliencia son dos constructos que es necesario desarrollar desde la primera infancia para una formación íntegra como ser humano social, activo y autosuficiente. Este estudio sirve como base para mejorar el conocimiento actual de este tema, sobre todo el de los docentes, de manera que sea de utilidad a la hora de concienciar de la importancia de su desarrollo dentro de las aulas. Además, sirve como incentivo para potenciar una evolución positiva en aquellos alumnos con niveles bajos de resiliencia y autoconcepto, mejorando el proceso de enseñanza-aprendizaje y proporcionando una educación de calidad.

\section{- Conflicto de intereses.}

Los autores declaran no tener ningún conflicto de intereses. 


\section{- Agradecimientos.}

Esta investigación ha sido realizada bajo el programa de becas FPU (Formación del Profesorado Universitario) financiado por el Ministerio de Educación cultura y deporte del gobierno de España, concedida a la primera autora, Cristina Pinel Martínez, con referencia FPU16/05225.

\section{REFERENCIAS}

Acevedo, V.E. y Restrepo, L. (2012). De profesores, familias y estudiantes: fortalecimiento de la resiliencia en la escuela. Revista Latinoamericana de Ciencias Sociales, Niñez y Juventud, 10, 301-319.

Andrade, J.A., Duffay, L., Ortega, P.A., Ramírez, E. y Carvajal, J.E. (2017). Autoestima y desesperanza en adolescentes de una institución educativa del Quindío. Revista Duazary, 14(2), 179-187. Doi: 10.21676/2389783X.1968.

Bernal, F. y Gálvez, F. (2017). Ajuste escolar y rendimiento académico y su relación con la motivación y el autoconcepto. Análisis de diferencias en muestra chilena y española. Revista de Orientación Educacional, 31 (60), $1-23$.

Bouillet, D., Ivanec, T.P. y Miljević, R. (2014). Preschool teachers' resilience and their readiness for building children's resilience. Health Education, 114(6), 435-450. Doi: 10.1108/HE-11-2013-0062.

Campos, S.R. y González, M.C. (2018). Resiliencia en la escuela: Un camino saludable. Apuntes de Ciencia y Sociedad, 8(1), 9-22. Doi: 10.18259/acs.2018002

Coetzee, S., Ebersöhn, L., Ferreira, R. y Moen, M. (2017). Disquiet voices foretelling hope: rural teachers' resilience experiences of past and present chronic adversity. Journal of Asian and African Studies, 52(2), 201-216. Doi: $10.1177 / 0021909615570955$.

Coppari, N., Barcelata, B.E., Bagnoli, L. y Codas, G. (2018). Efectos de la edad, el sexo y el contexto cultural en la disposición resiliente de los adolescentes de Paraguay y México. Revista de Psicología Clínica con Niños y Adolescentes, 5(1), 16-22. Doi: 10.21134/rpena.2018.05.1.2.

Costa, S., Barontini, M., Forcada, P., Carrizo, P. y Almada, L. (2010). Estrés psicosocial y baja resiliencia, un factor de riesgo de hipertensión arterial. Revista argentina de cardiología, 78(5), 425-431.

Costa, S. y Tabernero, C. (2012). Rendimiento académico y autoconcepto en estudiantes de educación secundaria obligatoria según el género. Revista Iberoameticana de Psicología y Salud, 3(2), 175-193.

Díaz, C. y Barra, E. (2017). Resiliencia y satisfacción laboral en profesores de colegios municipales y particulares subvencionados de la comuna de Machalí. Estudios Pedagógicos XLIII, 7 (1), 75-86. Doi: 10.4067/S0718-07052017000100005.

Esturgó, M.E. y Sala, J. (2010). Disruptive behaviour of students in primary education and emotional intelligence. Teaching and Teacher Education, 26(4), 830-837.

Fernández, A., Goñi, E., Rodríguez, A. y Goñi, A. (2017). Diferencias de sexo y edad en el autoconcepto social. Universitas Psychologica, 16(2), 192-201. Doi: 10.11144/Javeriana.upsy. 16-2.dsea.

Fínez, J. y Morán, C. (2016). La resiliencia y su relación con salud y ansiedad en estudiantes españoles. International Journal of Developmental and Educational Psychology, 1 (1), 409-416. Doi: 10.17060/ ijodaep.2015.n1.v1.90.

Fínez, M.J. y Morán, C. (2014). Resiliencia y autoconcepto: su relación con el cansancio emocional en adolescentes. International Journal of Developmental and Educational Psychology INFAD Revista de Psicología, 7 (6), 289-296. Doi: 10.17060/ijodaep.2014. n1.v6.746

Fínez, M.J. y Morán, C. (2017). Resiliencia y autovaloraciones esenciales: Estudio comparativo en adolescentes y jóvenes. Physchology. society and education, 9(3), 347-356. Doi: 10.25115/psye.v9i3.857

Fuentes, M., García, J., Gracía, E. y Lila, M. (2011). Autoconcepto y ajuste psicosocial en la adolescencia. Psicothema, 23(1), 7-12.

Ganotice, F.A., Yeung, S.S., Beguina, L.A. y Villarosa, J.B. (2016). In search for H.E.R.O among Filipino teachers: the relationship 
of positive psychological capital and workrelated outcomes. Asia-Pacific Education Researcher, 25(3), 407-414. Doi: 10.1007/ s40299-015-0267-9

Garaigordobil, M., Durá, A. y Pérez, J. (2005). Síntomas psicopatológicos, problemas de conducta y autoconcepto-autoestima: Un estudio con adolescentes de 14 a 17 años. Anvario de Psicología Clínica y de la Salud, 1, 53-63.

García, M.C.yDomínguez, E. (2013). Desarrollo teórico de la Resiliencia y su aplicación en situaciones adversas: Una revisión analítica. Revista Latinoamericana de Ciencias Sociales, Niñez y Juventud, $17(1), 63-77$. Doi: 10.11600/1692715x.1113300812

García, M., Ríos, M.l., Carrillo, C. y Sabuco, E. (2018). The moderating role of resilience in the relationship between academic burnout and the perception of psychological health in nursing students. Educational Psychology, 38(1), 1-13. Doi: 10.1080/01443410.2015.1044944

Gonzáles, E., Huamán, M., Cubas, N. y Visalot, G. (2018). Resiliencia y autoconcepto de los estudiantes, Facultad de Enfermería Universidad Nacional Toribio Rodríguez de Mendoza de Amazonas, Chachapoyas - 2014. Revista de Investigación Científica UNTRM: Ciencias de la Salud, 1 (1), 59-65. González, N.I. y Valdez, J.L. (2015). Resiliencia. Diferencias por Edad en Hombres y Mujeres Mexicanos. Acta de Investigación Psicológica, 5(2), 1996-2010. Doi: 10.1016/S2007. 4719(15)30019-3

Hernández, V., Hernández, M.S. y López, A. (2017). Uso de redes sociales y autoconcepto en niños de primaria. PsicoEducativa: reflexiones y propuestas., 3(5), 31-37.

Himmel, E. (2002). Modelos de análisis de la deserción estudiantil en la educación superior - Retención y movilidad estudiantil. Revista Calidad en la Educación, 17, 91 108.

Kim, S.R. y Lee, S.M. (2018). Resilient college students in school-to-work transition. International Journal of Stress Management, 25(2), 195-207. Doi: 10.1037/str0000060
Kong, F., Wamg, X., Hu, S. y Liu, J. (2015). Neural corelates of psychological resilience and their relation to life satisfaction in a sample of healthy young adults. Neuroimage, 123, 165-172. Doi: 10.1016/i.neuroimage.2015.08.020

Muñoz, V. (2016). La resiliencia: una intervención educativa en pedagogía. Revista nacional e internacional de educación inclusiva, 9(1), 79-89.

Padilla, M.T., García, S. y Suárez, M. (2010). Diferencias de género en el autoconcepto general y académico de estudiantes de $4^{\circ}$ de ESO. Revista de Educación, 352, 495 515.

Pintado, S. y Cruz, M.F. (2017). Factores de resiliencia en niños y adolescentes con cáncer y su relación con el género y la edad. CIENCIA ergo-sum, 24(2), 137 144.

Pinto, C.G. (2014). Psychological resilience: an approach to the concept, theoretical framework and relation with child sexual abuse. Summa Psicológica UST, $11(2)$, 19-33. Doi: https://doi. org/10.18774/448x.2014.11.129

Ramírez, I.A. y Castro, M. (2018). Análisis de los niveles de resiliencia en función del género y factores del ámbito educativo en escolares. ESHPA-Education, Sport, Health and Physical Activity., 2(1), 50-61.

Reynoso, O.U., Caldera, J.F., De la Torre, V., Martínez, A. y Macías, G. (2018). Autoconcepto y apoyo social en estudiantes de bachillerato. Un estudio predictivo. Revista de Psicología y Ciencias del Comportamiento, 9(1), 100-119. Doi: 10.29365/rpcc.20180529-66

Richards, A.R., Levesque, C., Templin, T.J. y Graber, K.C. (2016). The impact of resilience on role stressors and burnout in elementary and secondary teachers. Social Psychology of Education, 19(3), $511-536$. Doi: 10.1007/s $11218-016-9346-x$

Rincón, B. (2016). Resiliencia y educación social Resilience and social education. Revista Iberoamericana de Educación, 70(2), 79-94. 
Rodríguez, A., Ramos, E., Martínez, I. y Rey, A. (2018). Implicación escolar de estudiantes de secundaria: la influencia de la resiliencia, el autoconcepto y el apoyo social percibido. Educación XX1, 21(1), 87-108. Doi: 10.5944/EDUCXX1.16026

Salum, A., Marín, R. y Reyes, C. (2011). Relevancia de las dimensiones del autoconcepto en estudiantes de escuelas secundarias de Ciudad Victoria, Tamaulipas, México. Revista Electrónica de Psicología Iztacala, 14(2), 255-272.

Sampedro, P., Alvarez, P. y Begega, A. (2018). Coping with stress during aging: The importance of a resilient brain. Current Neuropharmacology, 16(3), 284-296. Doi: 10.2174/1570159X15666170915141610

Sosa, D., Sánchez, S. y Guerrero, E. (2016). Autoconcepto académico: modalidades de escolarización, repeticiones de curso y sexo. Campo abierto, 35(2), 69-82.
Tabernero, C., Serrano, A. y Mérida, R. (2017). Estudio comparativo de la autoestima en escolares de diferente nivel socioeconómico. Psicologia Educativa, 23(1), 9-17. Doi: 10.1016/i.pse.2017.02.001

Valenzuela, B. y López, M.D. (2015). Autoconcepto de estudiantes universitarios chilenos con discapacidad. Diferencias en función del género. Revista Nacional e Internacional de Educación Inclusiva, 8(3), 153-170.

Villalta, M. y Saavedra, E. (2012). Cultura escolar, prácticas de enseñanza y resiliencia en alumnos y profesores de contextos sociales vulnerables. Universitas Psychologica, 11 (1), 67-78. Doi: 10.11144/Javeriana.UPSY131 .oete

Vinaccia, S., Quiceno, J.M. y Moreno, E. (2007). Resiliencia en adolescentes. Revista Colombiana de Psicología, 16(1), 139-146. 